\title{
Vulnerability to torrent processes
}

\author{
S. Fuchs \\ Institute of Mountain Risk Engineering, \\ University of Natural Resources and Applied Life Sciences, Vienna, \\ Austria
}

\begin{abstract}
In natural hazards research, risk is defined as a function of (1) the probability of the occurrence of a hazardous process, and (2) the assessment of the related extent of damage, defined by the values at risk and the vulnerability according to the intensity of the hazard process. Until now, only a little work has been carried out to determine vulnerability values for objects exposed to torrent processes, in particular to debris flows. The vulnerability values proposed in the literature show a wide range, above all with respect to medium and high process intensities. Furthermore an application of these values might lead to an overestimation of vulnerability, as an assessment for alpine torrent events has shown. In this study, data from Austria and Switzerland were used to empirically analyse and assess the vulnerability of buildings to torrent processes, and to establish a respective vulnerability function. This function was found to be valid for debris flow intensities between $0.33 \mathrm{~m}$ and $3.06 \mathrm{~m}$, a typical range for debris accumulation on torrential fans in alpine catchments. Since the analysis was based on process intensities and is thus independent from recurrence intervals, not only the risk resulting from design events can be calculated but also every other event with a different frequency. A wider application of the developed method to additional test sites would allow for further improvement of the results and would support an enhanced standardisation of the vulnerability function, especially with respect to the possible risk-reducing effects of different mitigation measures.
\end{abstract}

Keywords: risk analysis, torrent processes, debris flows, vulnerability. 


\section{Introduction}

The term vulnerability is closely related to the consequences of natural hazards, and is used in hazard and disaster management in a large number of ways. These consequences are generally measured in terms of damage or losses, either on an ordinal scale based on social values or perceptions and evaluations, or on a metric scale (e.g., as a monetary unit). Consequently, two diverse perspectives on the concept of vulnerability exist; (1) the perspective from social sciences and (2) the perspective from natural sciences. Focussing on the latter, and thus neglecting any social implications arising from hazards, vulnerability is usually considered as a function of a given process intensity towards physical structures. Therefore, vulnerability is related to the susceptibility of elements at risk, and is defined as the expected degree of loss for an element at risk as a consequence of a certain event [1]. Consequently, vulnerability values range from 0 (no damage) to 1 (complete destruction). Its assessment usually involves the evaluation of several different parameters and factors such as building materials and techniques, state of maintenance, presence of protection structures, presence of warning systems and so on [2]. On the impact side, empirical process parameters such as the intensity have to be analysed based on theories of probability, which is usually undertaken by mapping the geomorphologic disposition and the extent of previous events, and by modelling (defined design) events.

Even though the latter perspective on vulnerability had been subject to extensive research and practical application in recent decades, considerable gaps still exist with respect to standardised equations allowing for a wider application of technical vulnerability assessments (e.g. [3]). This has to be attributed to the overall lack of data, in particular concerning losses caused by alpine natural hazards, which are often a result of missing empirical quantification. Recently, promising approaches for the quantification of vulnerability have been made with respect to avalanches and rock fall processes, respectively [4-6]. However, sound suggestions for landslides and torrent processes are still outstanding, even if these processes caused major losses worldwide as well as in European mountain regions in recent years. An overview concerning the current state of the art in vulnerability assessment for landslide risk focussing on torrent processes is provided by Fuchs et al. [7]. As a consequence of research design, individual approaches vary significantly in scale and resulting numerical values (table 1). Although vulnerability analysis is part of the consequence evaluation during a risk assessment procedure, many approaches neither specify the type of process they are applicable to (e.g., "landslides", debris flows, hyperconcentrated flows), nor the physical mechanisms (e.g., travel distance) or the structural resistance of elements at risk. In particular, information on process intensities is often missing and therefore a valuation is only carried out semi-quantitatively.

Thus, neither a unique method nor an overall applicable vulnerability function is currently available for the assessment of landslide risk, and in particular with respect to torrent processes or debris flows. The overall aim of the study presented here was to close this gap by providing analyses of vulnerability due to torrent events, and by contributing to a comprehensive vulnerability function. 
Table 1: Vulnerability values applicable to torrent-related processes.

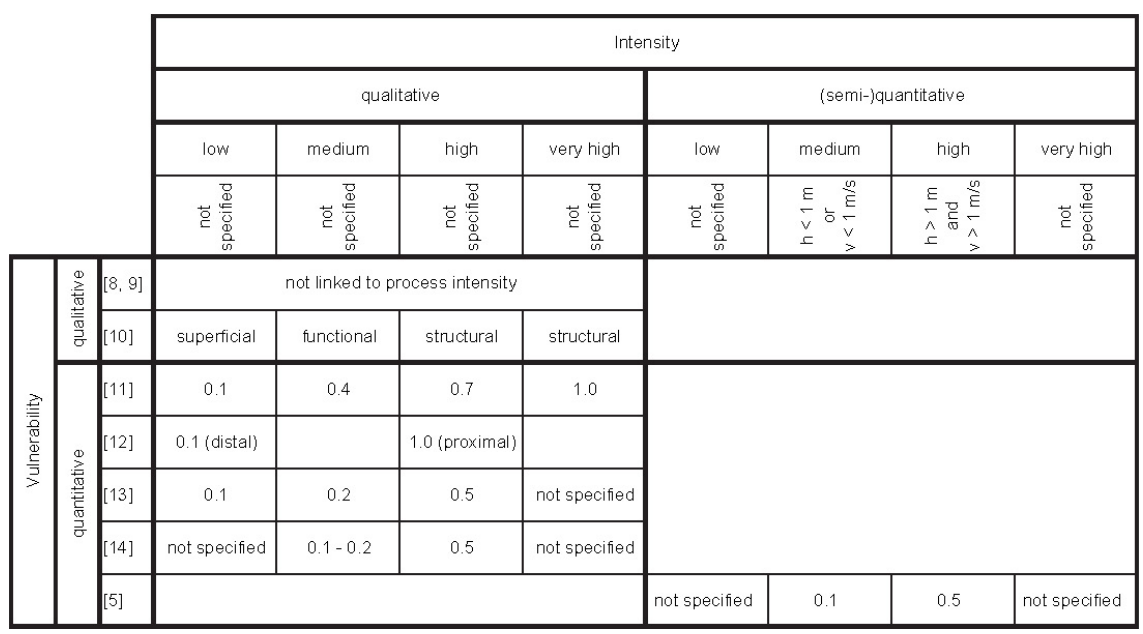

\section{Vulnerability assessment}

The study was carried out by analysing well-documented torrent events in the Eastern Alps, Austria. Two test sites were found to be most suitable for detailed case studies, (1) the Wartschenbach catchment concerning debris flow events and (2) the Vorderbergerbach catchment with respect to hyperconcentrated flows.

(1) The Wartschenbach catchment is situated in the Eastern Alps in the community of Nußdorf-Debant in the Drau valley, next to the city of Lienz, Austria, between $670 \mathrm{~m}$ and $2,113 \mathrm{~m}$ a.s.l. The geology is dominated by para-gneiss and mica schist; and covered by glacial deposits. Due to the considerable amount of unconsolidated material, and due to the steep gradient of $30-40 \%$, the catchment is susceptible to erosion processes, in particular debris flows. Several damaging torrent events are recorded in the event registry.

(2) The Vorderbergerbach catchment is the right tributary to the Gail river in the Carnian Alps, which represent the border with Italy in the Southern part of Carinthia, Austria. The catchment area covers $26 \mathrm{~km}^{2}$ between $690 \mathrm{~m}$ and $1,560 \mathrm{~m}$ a.s.l. Lithologically, the basin is comprised from limestone and Ordovician shale, and covered by deposits from the Wurmian glaciation. Several damaging torrent events are recorded in the event registry causing damage in the village of St. Stefan-Vorderberg located on the fan.

The vulnerability of elements at risk was measured using an economic approach. The main criterion therefore was the damage susceptibility (vulnerability), which describes the amount of damage related to the specific damage potential of the considered element at risk, often referred to as loss severity. Following this definition, vulnerability values were derived from the quotient between loss and individual reinstatement value for each element at risk. In a second set of 
calculations, these ratios obtained for every single building in the test site were attributed to the process intensities of respective hazard events. As a result, a vulnerability function was developed, linking process intensities to object vulnerability values. Consequently, this vulnerability function was used as a proxy for the structural resistance of buildings regarding dynamic debris flow impacts, and thus was used for a spatially explicit assessment of debris flow susceptibly.

The elements at risk were analysed with respect to their spatial location and extension using GIS. The size of the buildings was recorded from digital datasets of the communal administration and provided the basis for a monetary evaluation of the reconstruction values. These values were calculated using the volume of the buildings and average prices per cubic metre according to the type of building $[15,16]$. Different price levels were applied, depending on the function of the buildings as well as on the number and kind of storeys. This information was extracted from the construction descriptions and updated by field studies. Taking into account inflation, the current average reconstruction value for every building resulted.

The losses due to the events were collected using information from the federal authorities. Since in Austria an obligatory building insurance against losses from natural hazards is not available so far [7], property losses are partly covered by a governmental fund. Consequently, these losses are collected on an object level immediately after an event by professional judges. For this study, these data were adjusted to inflation and attributed to the information on every single element at risk using GIS.

The process characteristics in the accumulation areas were determined on the basis of process documentation carried out subsequently after the individual event by the Austrian Torrent and Avalanche Control Service, a federal institution operating throughout Austria to protect the population from torrents, erosion and avalanches. These data were supplemented by the analysis of data gathered from a re-calculation of the events, above all a reconstruction of the accumulation heights and flow depths on the torrential fans using the twodimensional simulation model FLO-2D (figure 1). Accumulation heights and flow depths were used as proxies for the process intensities in the accumulation areas. As a result, different process intensities were determined for the events, dividing the accumulation areas into sections with different process severities. According to this procedure, the intensity-vulnerability function is independent from recurrence intervals, and can therefore be applied to any other event with a different frequency.

\section{Results}

In figure 2, the empirical intensity-vulnerability relation is shown for detached family houses, the predominant type of building in the test sites. The prevailing construction is a brick masonry and concrete construction for the main floors and the cellar, respectively. The process intensity, plotted as the abscissa in terms of 


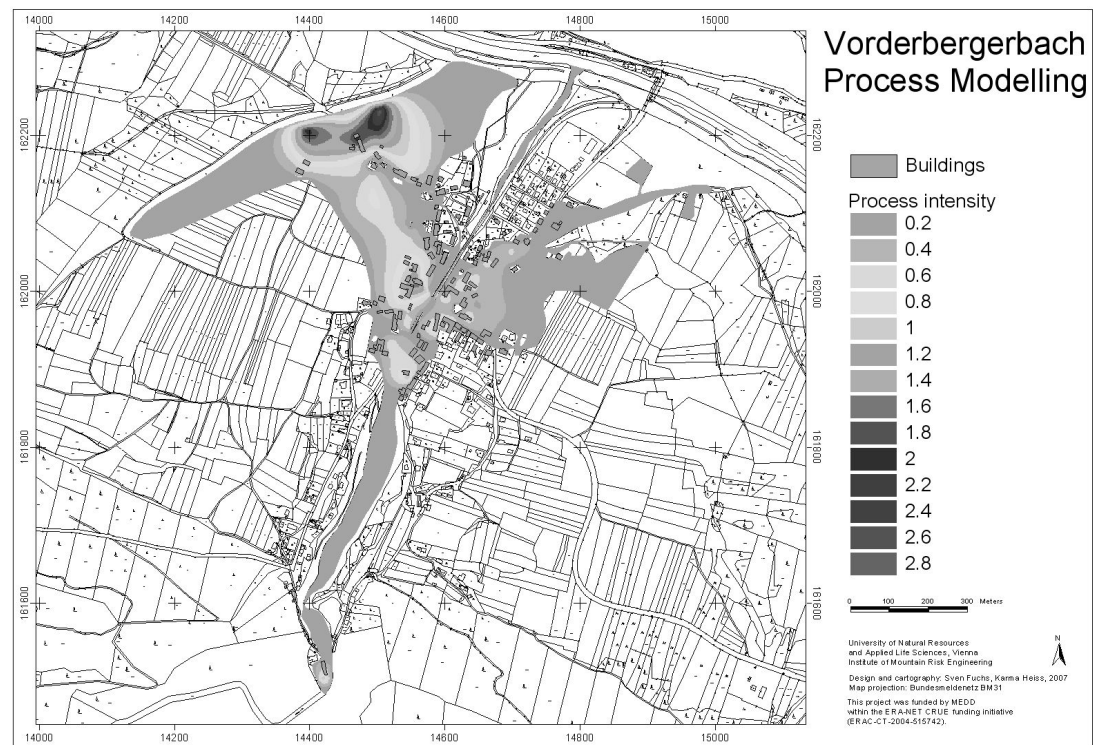

Figure 1: Reconstruction of accumulation heights and flow depths on individual torrent fans using the two-dimensional simulation model FLO-2D.

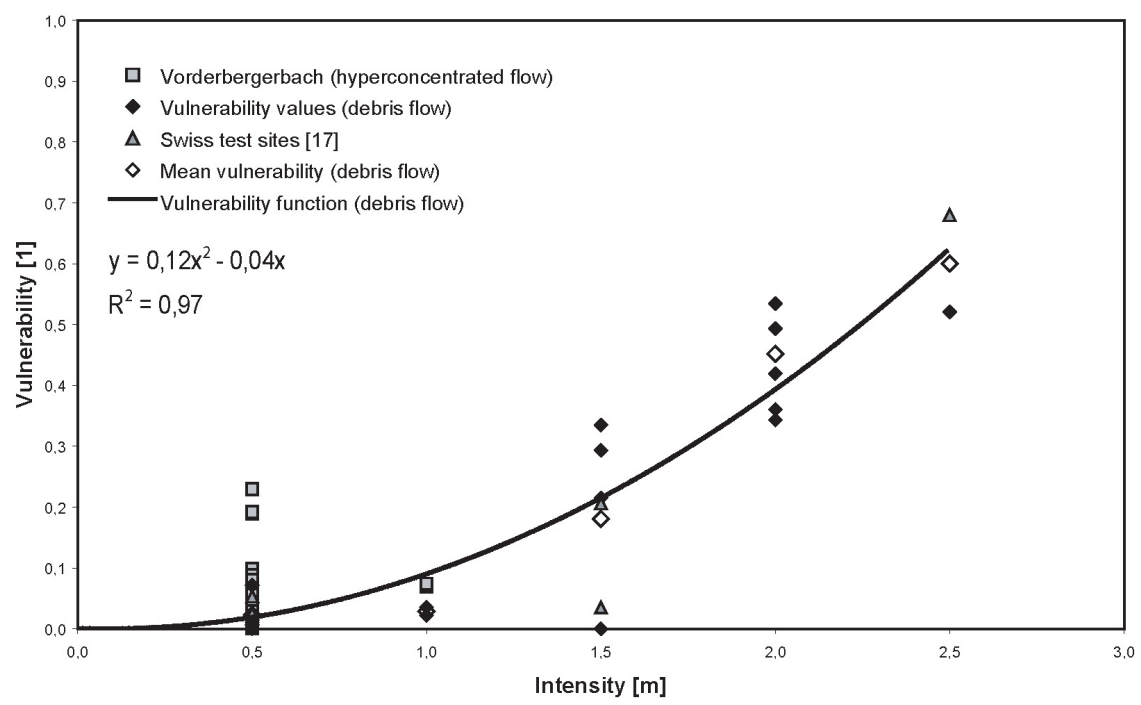

Figure 2: Vulnerability function for debris flows. Data related to debris flows is shown by solid black rhombi (the mean is shown by framed white rhombi). Data from Swiss test sites [17] is presented by grey triangles. Data originating from hyperconcentrated flows is shown by grey squares. For the calculation of the vulnerability function, data related to hyperconcentrated flows was neglected. 
deposit height, was grouped in steps of 0.5 metres. In general, the results suggest a low vulnerability if the process intensity is low and an increased vulnerability if the process intensity is higher. In detail, the data do not suggest a linear increase in vulnerability, which is a result of the specific process characteristics. Low process intensities cause noticeably less damage than medium and high intensities.

$$
f_{(x)}=\left\{\begin{array}{l}
0 \text { if } x<0 . \overline{3} \\
0.12 x^{2}-0.04 x \text { if } 0 . \overline{3} \leq x \leq 3.06 \\
1 \text { if } x>3.06
\end{array}\right.
$$

The relationship between debris flow intensity $\mathrm{x}$ and vulnerability $\mathrm{y}$ in the Wartschenbach area, supplemented by additional studies carried out in the Swiss Alps [17], was found to fit best to the data by a second order polynomial function for all intensities $0.33 \leq \mathrm{x} \leq 3.06 \mathrm{~m}$, see eqn. (1). The coefficient of determination $\mathrm{R}^{2}$ is 0.97 , which seems to be comparatively sound with respect to the amount of data available.

- Within the intensity class of 0.5 metres, the statistical spread of the vulnerability values is low (0.00-0.07), and the mean vulnerability is 0.02 .

- Within the intensity class of 1.0 metres, the statistical spread of the vulnerability values is low (0.02-0.04), and the mean vulnerability is 0.03 .

- Within the intensity class of 1.5 metres, the statistical spread of the vulnerability is remarkable $(0.00-0.33)$, and the mean vulnerability is 0.18 .

- Within the intensity class of 2.0 metres, the statistical spread of the vulnerability is again high (0.34-0.53), and the mean vulnerability is 0.45 .

- The intensity class of 2.5 metres is only applicable to two buildings, with a vulnerability of 0.52 and 0.68 , respectively; the resulting mean vulnerability is 0.6 . Even if it is due to limited data this value may presumably change if more records were available, a considerable increase in vulnerability is detectable in comparison to lower process intensities.

A process intensity of $0.33 \mathrm{~m}$ was found to represent a lower impact threshold since no damage to buildings occurred below this value. Taking into consideration the relatively formal procedure of applying for subsidies from the federal and national funds in Austria, this lower threshold might be an artefact since similar data from Italy had shown minor losses related to such process intensities [18].

In addition, the analysis of the data had shown that the vulnerability of buildings affected by medium debris flow intensities $(1.00-1.50 \mathrm{~m})$ is highly dependent on whether or not the entrained material harms the interior of the building (i.e., by an intrusion of material through openings such as doors, wells and windows). Consequently, local protection measures such as deflection walls and specially designed closure structures for at-grade openings definitely play a major role in reducing the vulnerability of buildings, particularly with respect to low and medium debris flow intensities [19].

Due to different process characteristics of debris flows and hyperconcentrated flows, the Vorderbergerbach data were not taken into account for the above- 
described vulnerability function. The results in the Vorderbergerbach area are shown in figure 2 by grey squares, and are characterised by a considerable range in vulnerability in the low-intensity sections. Within the intensity class of 0.5 metres, the spread of vulnerability is considerable high between 0.01 and 0.37 , the corresponding mean amounts to 0.08 ; and within the intensity class of 1.0 metres, the spread is low between 0.06 and 0.07 , with a mean of 0.07 . Hyperconcentrated flows seem to cause considerable losses already at low process intensities, which might be a result of the surplus of liquid fraction in the sediment.

\section{Discussion}

If risk analyses are carried out with respect to the probable maximum loss, a vulnerability value of 1 will generally be assigned to exposed elements at risk [3]. However, such solutions are not very valuable with respect to a better understanding of the vulnerability of elements at risk to torrent events. A general strategy in determining vulnerability of elements at risk to specific events is still missing. Until now, vulnerability models are mainly based on plausibility issues, expert knowledge, conceptual approaches, and assessments of historical data. Hence, they are for the most part based on qualitative statements on observed damage. Furthermore, this data is hardly transferable to future scenarios since the impact force of the process and thus the process intensity is not known.

In the previous section, an empirical vulnerability function for debris flows was presented for alpine test sites applicable to process intensities between $0.33 \mathrm{~m}$ and $3.06 \mathrm{~m}$. It had been shown that this function follows a polynomial distribution, which is consistent with recently published preliminary results [7]. However, by definition, vulnerability ranges from 0 and 1 . Consequently, for process intensities higher than approximately $3 \mathrm{~m}$, vulnerability cannot be satisfyingly mirrored by such a polynomial, because an overall vulnerability function has to fulfil the constraint shown in eqn. (2). On the other hand, such high process intensities generally result in a total loss of the building since the arising efforts to repair the damage will exceed the expenditures necessary for a completely new construction.

$$
\lim _{x \rightarrow \infty} f_{(x)}=1
$$

Vulnerability is needed during the legally prescribed procedure of cost-benefit analyses during the planning of protection measures in Austria. Hence the susceptibility of values at risk is a key parameter during the risk assessment procedure. The function presented in figure 2 shows considerable lower vulnerability values than the Swiss intensity-susceptibility relationship presented in table 1 [5]. Furthermore, for process intensities $<2.0 \mathrm{~m}$ the curve is running below the values suggested by the responsible Austrian Ministry for the consideration of buildings susceptibility during measurement planning [20], see table 2 . Moreover, the stepped increase in vulnerability provided by both studies 
Table 2: $\quad$ Vulnerability values suggested for use in Austria [20].

\begin{tabular}{|c|l|l|}
\hline Process intensity & \multicolumn{2}{|c|}{ Vulnerability } \\
\hline$<0.7 \mathrm{~m}$ & 0.1 (residential buildings) & 0.2 (hotel industry) \\
\hline$\geq 0.7 \mathrm{~m}$ & 0.3 (residential buildings) & 0.5 (hotel industry) \\
\hline
\end{tabular}

$[5,20]$ cannot be supported by the results presented above. As a result, the benefit-cost ratio calculated when applying the Swiss and Austrian guidelines, respectively, might result in an overestimation of benefit created by mitigation measures.

Vulnerability is highly dependent on the construction material used for exposed elements at risk. The buildings studied within the test site were constructed by using brick masonry and concrete, a typical construction design in post-1950s building craft in alpine countries. Consequently, the presented intensity-vulnerability relationship is applicable to this mixed construction type within European mountains. The presented method followed a spatial approach, and was based on process intensities, the volume of elements at risk and average reconstruction values in dependence of the surface area on an object basis. Since vulnerability was defined using an actuarial approach, the relation between reconstruction values and losses is principally applicable in regions with different economic background. However, an expansion of the presented method to additional test sites would allow for a further improvement of the results, above all an application to other buildings types. This would support an enhanced standardisation of the vulnerability function.

It had been shown in recent studies that temporal changes of risk levels in European mountain regions are considerable both, on a long-term and on a shortterm scale [21]. These changes result from the dynamics in every individual factor to be considered during the risk assessment procedure, i.e., the probability of occurrence of the hazardous process, the values at risk and the vulnerability. Apart from the question of what level of loss to expect, vulnerability tends to be a dynamic concept in relation to the perpetual duality between efforts to reduce or mitigate risks and human actions that create risks or increase their levels [22]. Viewed in terms of risk management, vulnerability of socio-economic systems to torrent events is a function of the costs and benefits of inhabiting mountain regions mediated by decisions taken on the basis of risk perception. As torrent risk is fundamentally a product of hazard, vulnerability and elements at risk, risk management issues from the point of view of social sciences and natural sciences should be combined for an efficient risk reduction. Hence, mechanisms of (intuitive or institutional) decision-making processes and functional relationships between individual factors have to be jointly combined for a sustainable risk management.

\section{References}

[1] Varnes, D., Landslide hazard zonation: A review of principles and practice, Paris, 1984. 
[2] Holub, M. \& Fuchs, S., Benefits of local structural protection to mitigate torrent-related hazards, this volume.

[3] Glade, T., Vulnerability assessment in landslide risk analysis, Die Erde, 134, pp. 123-146, 2003.

[4] Wilhelm, C., Wirtschaftlichkeit im Lawinenschutz. Mitteilungen SLF, 54, Davos, 1997.

[5] Borter, P., Risikoanalyse bei gravitativen Naturgefahren - Methode. Umwelt-Materialien, 107/II, ed. BUWAL, Bern, 1999.

[6] Barbolini, M., Cappabianca, F. \& Sailer, R., Empirical estimate of vulnerability relations for use in snow avalanche risk assessment, ed. Brebbia, C., Risk Analysis IV, Southampton, pp. 533-542, 2004.

[7] Fuchs, S., Heiss, K. \& Hübl, J., Towards an empirical vulnerability function for use in debris flow risk assessment, Natural Hazards and Earth System Sciences, 7, pp. 495-506, 2007, http://www.nat-hazards-earth-systsci.net $/ 7 / 495 / 2007 /$.

[8] Leone, F., Asté, J.-P. \& Leroi, E., L'évaluation de la vulnérabilité aux mouvements du terrain, Revue de Géographie Alpine, 84, pp. 35-46, 1996.

[9] Finlay, P., The risk assessment of slopes, PhD Thesis, School of Civil Engineering, University of New South Wales, Sydney, Australia, 1996.

[10] Cardinali, M., Reichenbach, P., Guzzetti, F., Ardizzone, F., Antonini, G., Galli, M., Cacciano, M., Castellani, M. \& Salvati, P., A geomorphological approach to the estimation of landslide hazards and risk in Umbria, Central Italy, Natural Hazards and Earth System Sciences, 2, pp. 57-72, 2002, http://www.nat-hazards-earth-syst-sci.net/2/57/2002/.

[11] Fell, R. \& Hartford, D., Landslide Risk Management, ed. Cruden, D. \& Fell, R., Landslide Risk Assessment, Rotterdam, pp. 51-109, 1997.

[12] Michael-Leiba, M., Baynes, F., Scott, G. \& Granger, K., Regional landslide risk to the Cairns community, Natural Hazards, 30, pp. 233-249, 2003.

[13] Bell, R. \& Glade, T., Quantitative Risk Analysis for Landslides - Examples from Bíldudalur, NW Iceland, Natural Hazards and Earth System Sciences, 4, pp. 117-131, 2004, http://www.nat-hazards-earth-syst-sci.net/4/117/ $2004 /$.

[14] Romang, H., Kienholz, H., Kimmerle, R. \& Böll, A., Control structures, vulnerability, cost-effectiveness - A contribution to the management of risks from debris torrents, ed. Rickenmann, D. \& Chen, C., Debris-flow hazards mitigation, Rotterdam, pp. 1303-1313, 2003.

[15] Keiler, M., Zischg, A. \& Fuchs, S., Methoden zur GIS-basierten Erhebung des Schadenpotenzials für naturgefahreninduzierte Risiken, ed. Strobl, J. \& Roth, C., GIS und Sicherheitsmanagement, Heidelberg, pp. 118-128, 2006.

[16] Kranewitter, H., Liegenschaftsbewertung, Wien, 2002.

[17] Kimmerle, R., Schadenempfindlichkeit von Gebäuden gegenüber Wildbachgefahren, Master Thesis, Philosophisch-naturwissenschaftliche Fakultät, University of Berne, Switzerland, 2002.

[18] Dall'Amico, M., personal communication, 2008. 
[19] Holub, M. \& Hübl, J., Local protection against mountain hazards - State of the art and future needs, Natural Hazards and Earth System Sciences, 8, in press.

[20] BMLFUW, Richtlinien für die Wirtschaftlichkeitsuntersuchung und Priorisierung von Maßnahmen der Wildbach- und Lawinenverbauung gemäß $§ 3$ Abs. 2 Z 3 Wasserbautenförderungsgesetz, Bundesministerium für Land- und Forstwirtschaft, Umwelt und Wasserwirtschaft, Wien, 2005.

[21] Fuchs, S. \& Keiler, M., Variability of natural hazard risk in the European Alps - Evidence from damage potential exposed to snow avalanches, ed. Pinkowski, J., Disaster management handbook, London, pp. 264-275, 2008.

[22] Alexander, D., Vulnerability to landslides, ed. Glade, T., Anderson, M. \& Crozier, M., Landslide hazard and risk, Chichester, pp. 175-198, 2005. 